OPEN ACCESS

Edited by:

Keisuke Yonehara,

Aarhus University, Denmark

Reviewed by:

Pierre-Yves Risold,

University of Franche-Comté, France

James A. Carr

Texas Tech University, United States

Manuel A. Pombal,

University of Vigo, Spain

${ }^{*}$ Correspondence: Ethan K. Scott ethan.scott@uq.edu.au

Received: 14 September 2017 Accepted: 20 December 2017 Published: 17 January 2018

Citation:

Heap LA, Vanwalleghem GC, Thompson AW, Favre-Bulle I, Rubinsztein-Dunlop H and Scott EK (2018) Hypothalamic Projections to the Optic Tectum in Larval Zebrafish.

Front. Neuroanat. 11:135. doi: 10.3389/fnana.2017.00135

\section{Hypothalamic Projections to the Optic Tectum in Larval Zebrafish}

\author{
Lucy A. Heap ${ }^{1}$, Gilles C. Vanwalleghem ${ }^{1}$, Andrew W. Thompson ${ }^{1}$, Itia Favre-Bulle ${ }^{2}$, \\ Halina Rubinsztein-Dunlop ${ }^{2}$ and Ethan K. Scott ${ }^{1,3 *}$
}

'School of Biomedical Sciences, The University of Queensland, St. Lucia, QLD, Australia, ${ }^{2}$ School of Maths and Physics, The University of Queensland, St. Lucia, QLD, Australia, ${ }^{3}$ The Queensland Brain Institute, The University of Queensland, St. Lucia, QLD, Australia

The optic tectum of larval zebrafish is an important model for understanding visual processing in vertebrates. The tectum has been traditionally viewed as dominantly visual, with a majority of studies focusing on the processes by which tectal circuits receive and process retinally-derived visual information. Recently, a handful of studies have shown a much more complex role for the optic tectum in larval zebrafish, and anatomical and functional data from these studies suggest that this role extends beyond the visual system, and beyond the processing of exclusively retinal inputs. Consistent with this evolving view of the tectum, we have used a Gal4 enhancer trap line to identify direct projections from rostral hypothalamus $(\mathrm{RH})$ to the tectal neuropil of larval zebrafish. These projections ramify within the deepest laminae of the tectal neuropil, the stratum album centrale (SAC)/stratum griseum periventriculare (SPV), and also innervate strata distinct from those innervated by retinal projections. Using optogenetic stimulation of the hypothalamic projection neurons paired with calcium imaging in the tectum, we find rebound firing in tectal neurons consistent with hypothalamic inhibitory input. Our results suggest that tectal processing in larval zebrafish is modulated by hypothalamic inhibitory inputs to the deep tectal neuropil.

Keywords: zebrafish, hypothalamus, tectum, superior colliculus, SPIM (selective plane illumination microscopy), optogenetics

\section{INTRODUCTION}

In vertebrates, the superior colliculus, or optic tectum, is a highly laminated structure located in the midbrain (Sparks, 1988; Robinson and McClurkin, 1989; Sparks and Hartwich-Young, 1989; Meek and Nieuwenhuys, 1998; May, 2006; Krauzlis et al., 2013). In mammals, the superior colliculus receives afferent inputs from multiple sensory regions of the brain, and contains intricate and overlapping topographic maps of the sensory world (Lane et al., 1973; Dräger and Hubel, 1976; Knudsen, 1982; Druga and Syka, 1984; Jay and Sparks, 1987; Sparks, 1988; Withington-Wray et al., 1990; King et al., 1996; Crish et al., 2003; Chabot et al., 2013). In contrast to mammals, amphibians and fish lack a visual cortex (Lázár, 1973; Streidter and Northcutt, 1989). Instead, they have a proportionally larger tectum that is hypothesized to carry out some of the visual processing that the cortex performs in mammals (Nevin et al., 2010; Orger, 2016). In teleost fish, tectal afferents arrive in the tectal neuropil, which comprises (from dorsal to ventral): the stratum fibrosum marginale (SM), which does not receive direct retinal inputs, the stratum opticum (SO), stratum fibrosum et griseum superficiale (SFGS), stratum griseum centrale (SGC) and the stratum album centrale and stratum griseum periventriculare (SAC/SPV; Vanegas et al., 1974; Meek, 1983; Sas and Maler, 1986; Meek and Nieuwenhuys, 1998). The laminae 
spanning from the SO to the SAC/SPV are demarcated by robust innervation from the axons of retinal ganglion cells (RGCs), which convey visual information to the tectum (Fiebig et al., 1983; Struermer, 1988; Streidter and Northcutt, 1989; Niell and Smith, 2005; Corbo et al., 2012; Udin, 2012). In contrast, the SM receives axonal projections from the torus longitudinalis (Meek and Schellart, 1978; Perry et al., 2010), and lacks direct retinal inputs. As in birds and mammals, this visual projection is highly topographically organized, and in fish arises solely from the contralateral eye (Struermer, 1988; Easter and Nicola, 1996; Niell and Smith, 2005; Kita et al., 2015). In a variety of adult fish, nonretinal tectal afferents have been described anatomically. These have been shown to come from a variety of neural structures including the pretectum, the anterior and ventromedial thalamic nuclei (Northcutt, 1982; Fiebig et al., 1983; Meek and Nieuwenhuys, 1998), the hypothalamus (Amemiya, 1983; Fiebig et al., 1983; de Arriba and Pombal, 2007), the torus longitudinalis, torus semicircularis and nucleus ruber (Northcutt, 1982; Meek and Nieuwenhuys, 1998; Xue et al., 2001; Fame et al., 2006; Folgueira et al., 2007), as well as from hindbrain structures including the nucleus isthmi, reticular formation, dorsal funicular nucleus, eurydendroid cells in the cerebellum, and the trigeminal nuclei (Northcutt, 1982; Fiebig et al., 1983; Meek and Nieuwenhuys, 1998).

In zebrafish, the tectum's fundamental structure and cellular composition form early in development. In larvae at 3 days post fertilization (dpf), RGC axons begin arriving and the tectal neuropil's laminae have formed, as has the densely populated periventricular layer (PVL; Struermer, 1988). Nonretinal projections from the Raphe nucleus (Yokogawa et al., 2012; Filosa et al., 2016) and cerebellum (Heap et al., 2013) also innervate the neuropil. The tectal neuropil contains axons of these afferent structures, the dendrites of PVL neurons, and the axons of PVL interneurons. It is also sparsely populated with GABAergic superficial inhibitory neurons (SINs) that are located in the SO, and that have been described both anatomically and functionally (Del Bene et al., 2010; Robles et al., 2011; Dunn et al., 2016). PVL neurons are morphologically diverse, including both tectal interneurons and projection neurons (Scott and Baier, 2009; Robles et al., 2011). The tectal circuits arising from these cells are necessary for high-acuity vision (Gahtan et al., 2005), and for distinguishing between small prey items and larger visual features that may represent predators (Del Bene et al., 2010; Preuss et al., 2014; Semmelhack et al., 2014; Bianco and Engert, 2015; Dunn et al., 2016). Anatomical and functional studies have suggested that visual information principally enters the SO of the neuropil, and is progressively filtered by SINs and then PVL interneurons, before being relayed to other brain regions by the PVL projection neurons, the dendrites of which occupy the deep sublaminae of the neuropil (Scott and Baier, 2009; Del Bene et al., 2010; Robles et al., 2011; Gabriel et al., 2012; Preuss et al., 2014; Semmelhack et al., 2014; Barker and Baier, 2015; Temizer et al., 2015).

Inputs from the Raphe and cerebellum notwithstanding, the larval zebrafish tectum is viewed as a dominantly retinorecipient structure that is involved almost exclusively in visual processing. The list of described nonretinal inputs remains short in comparison to the diverse inputs received by the tectum in adult fish and the superior colliculus in mammals and birds. Nonetheless, larval zebrafish show behaviors that imply the integration of visual input with more complex state traits such as hunger (Filosa et al., 2016), and tectal neurons respond to auditory and water-flow stimuli (Thompson et al., 2016; Vanwalleghem et al., 2017). This implies that the larval zebrafish's tectum has more numerous and diverse inputs, and more nuanced circuitry, than has thus far been described.

Decisions based on the metabolic state of an animal are largely driven by the hypothalamus, a region of the brain that controls the metabolic and endocrine processes through the hypothalamic-pituitary-adrenal axis (Smith and Vale, 2006; Ulrich-Lai and Herman, 2009). In mammals, the hypothalamus has been shown to play a role in a multitude of such behaviors (Kokoeva et al., 2005; Bolborea and Dale, 2013), and elements of the underlying circuitry have been described. These include inhibitory hypothalamic projections to the intermediate laminae of the superior colliculus, which are hypothesized to assist in the role that the superior colliculus plays in visual attention tasks (Pityk, 1979; Rieck et al., 1986; Gandhi and Katnani, 2011).

Recently, contributions of the hypothalamus to behaviors in larval zebrafish have been described. Populations of dopaminergic hypothalamic neurons have been shown to regulate light seeking and motor behaviors (Fernandes et al., 2012; McPherson et al., 2016), feeding (Yokobori et al., 2011, 2012) and sleep cycles (Chiu and Prober, 2013). Additionally, serotonergic neurons in the Raphe nucleus, which are targeted by hypothalamic neurons, have been shown to work with visual information to mediate the classification of visual stimuli as either appetitive or predatory based on the feeding state of an individual animal (Filosa et al., 2016).

In larval zebrafish, hypothalamic nuclei are not yet spatially differentiated; instead, the expression of numerous hypothalamic neuropeptides allow for the general identification of hypothalamic nuclei (Herget et al., 2014). On this basis, the homologs of mammalian hypothalamic nuclei have been identified in larval zebrafish, including the paraventricular nucleus and preoptic area (Herget et al., 2014), and the dopaminergic A11 group and subpallial dopaminergic neural populations (Tay et al., 2011).

Combined, the anatomical and functional connections that have been described in adult fish, and tetrapods, the functions that the hypothalamus plays in larval zebrafish, and the flexibility of tectal responses to visual stimuli, suggest that the hypothalamus may be influencing tectal activity directly or indirectly in larval zebrafish. In this study, we have used a transgenic Gal4 line with expression in the hypothalamus to map previously undescribed projections into the tectal neuropil of zebrafish larvae, and to identify the laminae and sublaminae of the tectal neuropil in which these projections terminate. We have then used optogenetics and sculpted light to drive activity selectively in the hypothalamus while performing calcium imaging in the tectal PVL, thus identifying 
the nature and magnitude of the hypothalamus' influence on tectal activity.

\section{MATERIALS AND METHODS}

\section{Generation of Animals}

All experiments were performed with approval from and in accordance with the University of Queensland Animal Welfare Unit (approval SBMS/378/16). Adult zebrafish (Danio rerio) were housed in a commercial RAS aquarium (Tecniplast S.p.A., Varese, Italy), in $28^{\circ} \mathrm{C}$ water $(\mathrm{pH} 7.5 \pm 0.26$, conductivity $992 \pm 35 \mu \mathrm{S} / \mathrm{cm}^{2}$, Ammonia 0-0.25 ppm, GH $83 \pm 25 \mathrm{ppm}$, $\mathrm{Ca} 2+76 \pm 26 \mathrm{ppm})$ on a 14:10 h light:dark photoperiod, with $310,000 \mu \mathrm{Js} / \mathrm{cm}^{2} \mathrm{UV}$ disinfection. Fish were fed twice daily with a mixed commercial diet of O.range Wean and NRD at a 1:1 ratio, with a dusting of Spirulina powder $(100 \mathrm{~g} / \mathrm{kg}$; INVE Aquaculture Thailand) at a rate of approximately $5 \%$ body mass per day. Fish were housed at a stocking density of 10 fish per liter. Larval fish were reared in a 3 per-ml rotifer polyculture, based on the method described in Best et al. (2010), before being weaned to an exclusive dry food diet by $30 \mathrm{dpf}$. Adult zebrafish were mated as previously described to generate larvae for experiments (Westerfield, 2000). All experiments were performed in animals homozygous for the nacre mutation of the Tupfel long fin (TLN) strain (Lister et al., 1999).

The ChR2(ET/TC)-mCherry plasmid (Berndt et al., 2011) was provided by K. Deisseroth (Stanford University). All subcloning for transgenesis was performed using the Gateway Tol2 transgenesis system (Kwan et al., 2007). pME-MCS (construct 237, Tol2kit v1.2) was altered to include additional cloning sites with the forward primer CCCGGGACCGGTAGATCTTGATCAGGATCC and the reverse primer GGATCCTGATCAAGATCTACCGGTCCCGGG, creating the plasmid pME-MCS_linker. ChR2(ET/TC)-mCherry was cloned into the middle entry vector pME-MCS_linker using a blunt ApaI and XbaI sites, creating PME_ChR2(ET/TC)mCherry. This was combined with a $10.5 \mathrm{X}$ UAS $5^{\prime}$ entry vector (construct 327, Tol2kit v1.2), a $3^{\prime}$ polyA containing vector (construct 302, Tol2kit v1.2) and a pDestTol2pA2 destination vector (construct 394, Tol2kit v1.2) using LR Clonase II Plus (Life Technologies) in a multi-site Gateway reaction. This generated the plasmid pDest_10.5XUAS:ChR2(ET/TC)-mCherry, which was confirmed by sequencing (AGRF, The University of Queensland, St. Lucia, QLD, Australia).

For transgenesis, plasmids were injected into single cell embryos within $40 \mathrm{~min}$ of fertilization, with an injection mix containing $75 \mathrm{ng} / \mu \mathrm{L}$ Tol2 transposase RNA and $100 \mathrm{ng} / \mu \mathrm{L}$ plasmid DNA. Injections were carried out in embryos obtained from crosses where a Gal4 ${ }^{\text {s1168t }}$;UAS:Kaede (Scott and Baier, 2009) animal was mated to an animal homozygous for the nacre mutation (Lister et al., 1999). Injected embryos were screened for transient expression of the protein at $48 \mathrm{~h}$ post fertilization, and were then raised to adulthood. Founders with offspring containing the desired transgene were identified using fluorescence microscopy, and were then outcrossed to TLN animals to create stable transgenic lines.

\section{Generation and Analysis of Averaged Transgene Expression Data for the Z-brain Atlas}

Animals expressing UAS:Kaede under the control of the Gal4 ${ }^{\text {s1113t }}$ transgene (Scott et al., 2007) were fixed, stained with the tERK antibody (Cell Signaling, ID 4696), and imaged as previously described (Randlett et al., 2015). Multiple tiles were stitched using the Pairwise Stitching ImageJ plugin (Preibisch et al., 2009). Image registration of Kaede expression was performed against a model of anti-tERK expression in the nervous system of larval zebrafish. This was performed with $\mathrm{CMTK}^{1}$ using the command string -awr 010203 - T 8 -X 52 -C 8 -G 80 -R 3 -A “--accuracy 0.4” -W “--accuracy 1.6”. Multiple $(n=9)$ registered animals were combined to create an average model of Kaede expression in Gal4 ${ }^{\text {s1113t }}$;UAS:Kaede animals, which was incorporated into a local version of the Z-Brain atlas. Analysis of the location of expression of a given transgenic line was performed using the Z-Brain toolbox (Randlett et al., 2015).

\section{Confocal Microscopy}

Animals expressing the desired fluorescent proteins, under the control of the Gal $4^{\text {s1113t }}$ were mated and raised as outlined above. All Kaede photoconversion experiments were carried out in $6 \mathrm{dpf}$ animals, which were screened for desired fluorescence at $2 \mathrm{dpf}$ and raised in the dark to avoid unwanted photoconversion. At $6 \mathrm{dpf}$, animals were mounted dorsal side down in 2\% low melt agarose (Progen Biosciences, Murrarie, QLD, Australia) in $50 \mathrm{~mm}$ glass bottom dishes (MatTek Corporation, Ashland, MA, USA), which were then filled with E3 media.

Photoconversions were performed on an Olympus BX61 upright confocal microscope, using $405 \mathrm{~nm}$ light focused into a $10 \mu \mathrm{m}$ region of interest (ROI), which was scanned across the cell bodies of neurons expressing UAS:Kaede. After conversions, animals were left for $1 \mathrm{~h}$ at $28.5^{\circ} \mathrm{C}$ in the dark, to allow photoconverted Kaede to diffuse from converted somae into axons. Fish were then imaged on an inverted Yokogawa 3i spinning disc confocal, using a $488 \mathrm{~nm}$ laser to image unconverted (green) Kaede, and a $561 \mathrm{~nm}$ laser to image converted (red) Kaede. Z-stacks were taken at $40 \times$ magnification, with a $0.2 \mu \mathrm{m}$ slice interval.

Other fluorescent microscopy of the Gal $4^{\text {s1113t }}$ line was performed on a Zeiss-LSM 710 inverted confocal microscope, using a $561 \mathrm{~nm}$ laser to image red fluorescence, and a $488 \mathrm{~nm}$ laser to image green fluorescence, and using either $10 \times$ or $20 \times$ objectives.

\section{Deconvolution}

Deconvolution of images acquired using the spinning disc confocal was performed with Huygens Professional Plus Deconvolution (Scientific Volume Imaging, Hilversum, Netherlands). A theoretical point spread function (PSF) was used, calculated from the parameters used for image acquisition. The signal to noise ratio (SNR) was calculated separately for each channel for each image by comparing the fluorescence intensity of a ROI to the fluorescence intensity

\footnotetext{
${ }^{1}$ http://www.nitrc.org/projects/cmtk/
} 
of the background. Deconvolution was performed with a total image change threshold of 0.01 , with single block processing on and a maximum iteration value of 60 .

\section{Spatial Analysis}

Spatial analysis of neuropil laminae was performed using Imaris v8.1 (Bitplane, Zurich, Switzerland). Surfaces of both the red and green channels in the neuropil were created using the "surface creation" plugin, where the neuropil was highlighted as a ROI. To remove any out-of-focus light remaining after deconvolution, a background subtraction of $0.2 \mu \mathrm{m}$ was used. Thresholding of the image was performed so that only axons in the neuropil were included in the surface reconstruction. Once surfaces were completed, clipping planes were used to section out a $20 \mu \mathrm{m}$ slice through the medial region of the rostral-caudal axis. To calculate the average intensity of the red and green channels, measurements of the fluorescence intensity was taken in three evenly spaced points, which were averaged to get the mean fluorescent intensity over the depth of the neuropil.

\section{Optogenetic Experiments}

Animals used for optogenetic experiments were generated by crossing animals carrying Gal4 ${ }^{\text {s1113t }}$ to animals carrying Gal4 $^{\text {s1168t }}$;UAS:ChR2-mCherry, HuC:H2B-GCaMP6s, creating larvae with the genotype Gal4 ${ }^{\text {s1113t }}$;UAS:ChR2-mCherry, $H u C: H 2 B-G C a M P 6 s$. Animals were screened for the desired fluorescent pattern at $2 \mathrm{dpf}$, and were raised until $6 \mathrm{dpf}$ as described above. Animals were mounted dorsal side up in $2 \%$ low melt agarose and immobilized using $100 \mu \mathrm{M}$ tubocurarine (tubocurarine hydrochloride pentahydrate, Sigma-Aldrich). Larvae were mounted in a custom built glass sided imaging chamber and were allowed to acclimate for $30 \mathrm{~min}$ prior to imaging on a house-built selective planar illumination microscope (SPIM; Thompson et al., 2016). Optogenetic experiments were performed by splitting a $488 \mathrm{~nm}$ laser between a spatial light modulator (SLM; HOLOEYE Photonics, Germany) and the illumination tube of the SPIM. To avoid off target optogenetic activation of ChR2, a 0.975 neutral density filter was added to the SPIM path upstream of the beam expander.

Imaging was performed at $5 \mathrm{~Hz}$, for $70 \mathrm{~s}$ (350 time points), at a depth of $50 \mu \mathrm{m}$ under the skin of the animal, with imaging focused on the tectum. The activation of the hypothalamic nuclei was performed at time points 50, 150 and 250, and with pulses lasting for either $100 \mathrm{~ms}$ for "short" experiments, or $5000 \mathrm{~ms}$ for "long" experiments. The hologram displayed on the SLM was iteratively calculated using the Gerchberg-Saxton algorithm (Gerchberg and Saxton, 1972; Whyte and Courtial, 2005), resulting in a $2 \mathrm{D}$ illumination source at a chosen depth. In relatively low-scattering medium we theorized that a $10 \mu \mathrm{m}$ disc would be very thin (Lutz et al., 2008), however by scanning the $10 \mu \mathrm{m}$ disc at the depth of the hypothalamus $(125 \mu \mathrm{m}$ below the skin), we determined that the light spread in the z-plane was approximately $15 \mu \mathrm{m}$ above and below the focal point (FavreBulle et al., 2015). Sibling controls not expressing ChR2 were subjected to identical experimental conditions to control for illumination.

\section{Optogenetic Image Analysis}

For movies taken during optogenetic experiments, we first deleted all frames in which the SLM was active; this resulted in movies containing frames 1-50, 77-150, 177-250 and 277-350 for long SLM experiments and frames 1-50, 53-150, 153-250 and 253-350 for short SLM experiments. This was done to avoid artifacts produced by the reflected SLM light. Images were then registered to eliminate drift in the $\mathrm{X}$ - and $\mathrm{Y}$-axes using the "Align_slices_in_stack" ImageJ plugin ${ }^{2}$. To create an individual ROI for each cell in the PVL and neuropil, an average Z-projection of the image sequence was generated and then cropped to the border of the PVL and neuropil. A mask of this image was created using the Morphological Segmentation Plugin in ImageJ with a watershed function tolerance of 18 (Meyer and Beucher, 1990; Legland et al., 2016). Oversegmenting was tolerated, as the merging of erroneously split cells was performed during subsequent MATLAB analysis of these data.

Analysis of all data was performed using a custom written MATLAB code. Data were imported as a 16 BIT .tiff series, which were then transformed into a 2-dimensional data matrix, containing the gray values of every pixel in the 350 -frame time series. Using the mask created above, the mean values of all pixels within each ROI were calculated for each time point. To measure the activity of individual neurons, the baseline fluorescence for each ROI was calculated by averaging the first ten time points for each individual ROI (F0). The raw gray value of every time point (FI) minus the baseline, was then divided by the baseline giving us the fluorescent change over time, which was then multiplied by 100 to give us percentage change over time:

$$
\Delta \mathrm{F} / \mathrm{F}=((\mathrm{FI}-\mathrm{F} 0) / \mathrm{F} 0) * 100
$$

After the $\Delta \mathrm{F} / \mathrm{F}$ of each ROI was calculated, ROIs with a correlation coefficient of above 0.97 were removed from the data using the corrcoeff MATLAB function. After duplicates had been removed, for each ROI at each SLM event, neural activity resulting from the SLM was identified by calculating the correlation of four time points before the SLM, and six time points after the SLM to three model profiles of GCaMP events. These were calculated by averaging 50 individual GCaMP signals over five separate movies (10 per movie) where cells were qualitatively excited or inhibited by the SLM illumination. For an ROI to be deemed either excited or inhibited, the minimum Pearson's correlation coefficient of all three SLM events to a model GCaMP profile had to be greater than 0.6 , and the maximum probability value was required to be below 0.001 . Every ROI that passed the above criteria was included as either an excited or an inhibited ROI, depending on which model spike it was correlated to. ROIs that did not meet these criteria were not included in the analysis. This analysis was performed on all experimental and control data. To compare the number of active cells between groups, an unpaired Student's $t$-test was performed (significance $<0.05$ ), as data were normally distributed (one sample $t$-test).

\footnotetext{
${ }^{2}$ https://sites.google.com/site/qingzongtseng/template-matching-ij-plugin
} 


\section{RESULTS}

\section{The Transgenic Line Gal4s1113t Expresses} Gal4 in Rostral Hypothalamic Neurons with Projections to the Tectal Neuropil

Through preliminary screening of an existing collection of Gal4 enhancer trap lines (Scott et al., 2007; Scott and Baier, 2009), we identified the transgenic line Gal4 $4^{\text {s1113t }}$ as containing apparent projections into the tectal neuropil in $6 \mathrm{dpf}$ larvae. An initial assessment of the anatomy of the line was performed using the transgenic line UAS:Kaede (Scott et al., 2007). Animals with the genotype Gal4 ${ }^{\text {s1113t }}$;UAS:Kaede were used to create a model of the average expression pattern of the Gal4 $4^{\text {s1113t }}$ transgenic line, which was then registered against the annotated Z-Brain atlas of the zebrafish brain (Randlett et al., 2015), and also compared with the the Atlas of Early Zebrafish Development (Mueller and Wullimann, 2005). Assessment against the Zebrafish Brain Atlas suggested expression within a small number of neurons in the vicinity of the rostral hypothalamus $(\mathrm{RH})$, with sparse labeling of neurons in the rhombencephalon and telencephalon (Figure 1). Neurites originating from the labeled neurons in the vicinity of the $\mathrm{RH}$ were seen in the midbrain and hindbrain (Figure 1H). The most notable concentration of neurites was found in the tectal neuropil, where Kaede was concentrated toward the medial (and therefore, deep) laminae. Outside of the vicinity of the
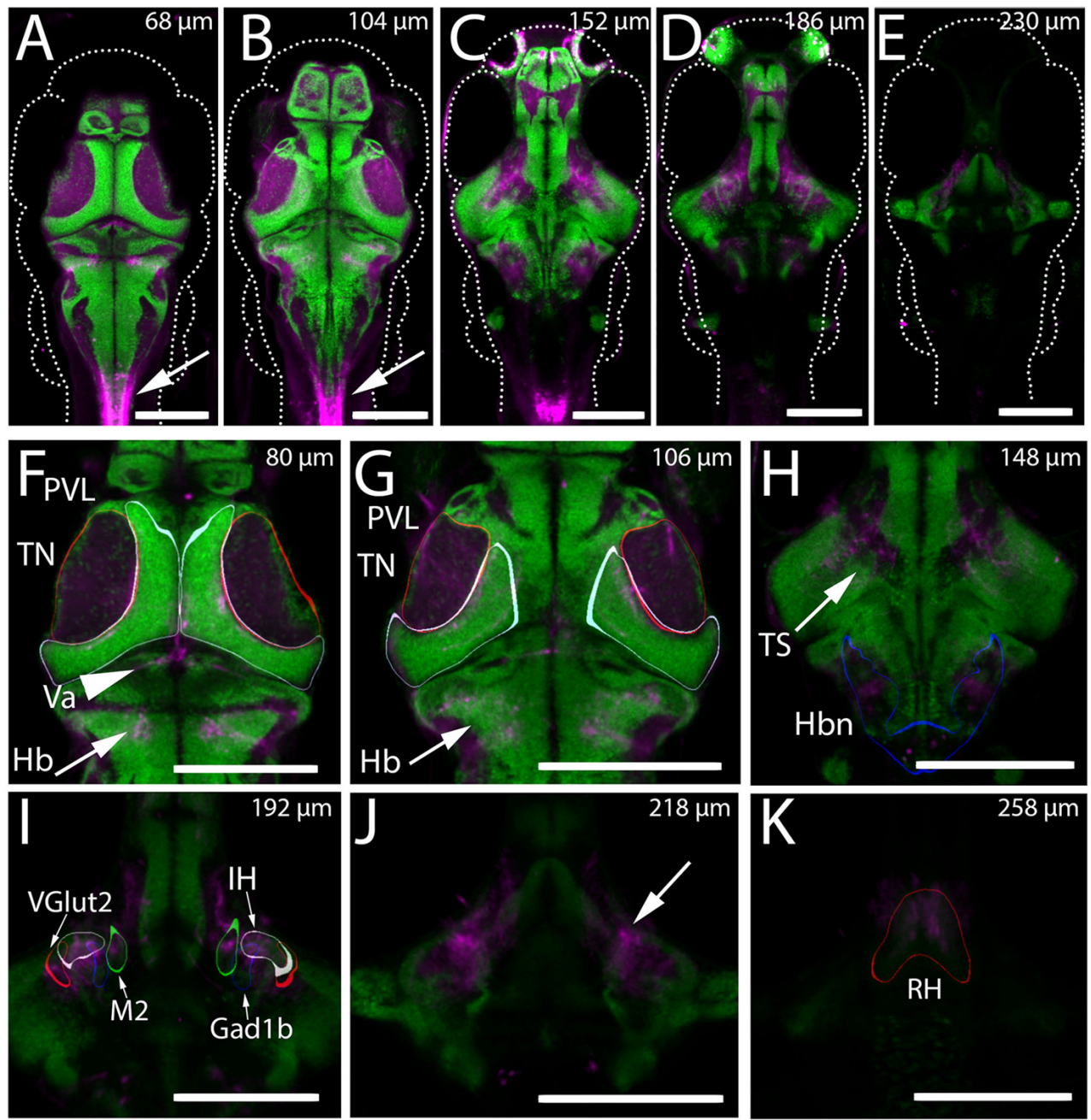

FIGURE 1 | Expression of Kaede in the Gal4 ${ }^{\text {s1113t }}$ ET line. (A-K) The mean intensity, resulting from registering and averaging the expression pattern across nine animals, of the genotype Gal4s1113t; UAS:Kaede is shown in magenta, overlaid with a pan-neuronal (HuC) H2B-RFP label (green). (A-E) Whole brain images at five dorsal-ventral depths separated by 15-21 microns. Arrows in (A,B) indicate expression in the spinal cord of these animals. (F) In the dorsal brain, Ga/4s1113t axons are present in the tectal neuropil (TN; red outline), tectal periventricular layer (PVL; cyan outline), valvula (Va) cerebellum (arrowhead) and hindbrain (Hb; arrow). (G) Axonal expression in the tectal neuropil (red outline) and sparse expression is seen in tectal periventricular neurons (cyan outline). Axons are present in the hindbrain (arrows). (H) Further ventral, expression is seen in the neuropil areas medial to the torus semicircularis (arrow) and in hindbrain neuropil (Hbn) regions (blue outline). (I) Axonal expression in the diffuse nucleus of the intermediate hypothalamus (IH; gray outline), a hypothalamic Gad1b cluster (blue outline) and hypothalamic Vglut2 cluster (red outline), and the migrated posterior tubercular area (M2; green outline). (J) Axonal labeling (arrow) of neurons with cell bodies located in the rostral hypothalamus $(\mathrm{RH}$; outlined in red in $\mathbf{K})$. Scale bars equal $200 \mu \mathrm{m}$. 
$\mathrm{RH}$, we observed labeled neurites in the valvula cerebellum (Figure 1F), within neuropil regions of the hindbrain caudal to the cerebellum, and also in a region medial to the torus semicircularis (Figure 1H). Observations of Kaede expression in individual Gal4 ${ }^{\text {s1113t }}$; UAS:Kaede animals supported these conclusions (Figure 2).

Overall, this analysis suggests that the majority of cell bodies labeled within Gal4 ${ }^{\text {s1113t }}$ are located within the vicinity of the $\mathrm{RH}$, with additional sparse labeling in the forebrain and hindbrain (Figures 2A,B). This, in turn, implies that the neurites visualized in these animals likely belong to these RH neurons. To confirm that the neurites observed in the tectal neuropil are sending synapses to the neuropil, we created Gal4 ${ }^{\text {s1113t; }}$ UAS:synaptophysin-GFP larvae (Heap et al., 2013; Hines et al., 2015), in which the presynaptic terminals of Gal4-positive neurons are labeled. Following photoconversion of Kaede in the whole animal, we observed dense GFP labeling of presynaptic terminals throughout the deep laminae of the tectal neuropil (Figure 2C), suggesting that these neurites are axons, and that they are forming synapses in, rather than simply passing through, the tectal neuropil.

\section{Hypothalamic Output Targets Specific Laminae of the Tectal Neuropil}

The above data establish that neurons expressing Gal4 under the control of Gal4 $4^{\text {s1113t }}$ transgene project axons into the tectal neuropil, but they do not conclusively demonstrate that the Gal4-positive RH neurons are the source of those axons. To address this, we performed targeted photoconversion of Kaede in the RH of Atoh7:Gal4; Gal4 ${ }^{\text {s1113t }}$, UAS:Kaede larvae, which express Kaede both in RGCs and throughout the Gal4 ${ }^{\text {s1113t }}$ expression pattern (arrow, Figure 3A). Following targeted photoconversion of $\mathrm{RH}$ neurons, red Kaede diffused down the axons of these neurons, arriving in the deep sublaminae of the tectal neuropil (Figure 3B). Kaede in RGC axons remained unconverted, assuring that off-target photoconversion was negligible (Figures 3B,C). Combined with the previous data, this confirms that the Gal4-positive neurons in the $\mathrm{RH}$ are the source of the observed presynaptic terminals in the tectal neuropil.

In light of this, and since functionally distinct laminae are an important part of tectal visual processing, we next undertook a detailed analysis of the neuropil laminae into which the $\mathrm{RH}$ axons project. The tectal neuropils of Atoh7:Gal4; Gal4 ${ }^{\text {s1113t }}$, UAS:Kaede larvae with targeted $\mathrm{RH}$ photoconversion contain both the axons of RGCs (green, labeling the SO, SFGS, SGC, and SAC/SPV), and the axons of $\mathrm{RH}$ projection neurons (containing photoconverted red Kaede). This provides a scaffold in green that allows us to register our $\mathrm{RH}$ afferents against the retinorecipient laminae of the neuropil. The strongest signal from $\mathrm{RH}$ projection neurons was in the deepest neuropil lamina: the SAC/SPV (arrows, Figures 3C-F). Other RH projections were present in the SFGS (arrowhead, Figures 3C,D,F), and a non-retinorecipient sublamina located between the SGC
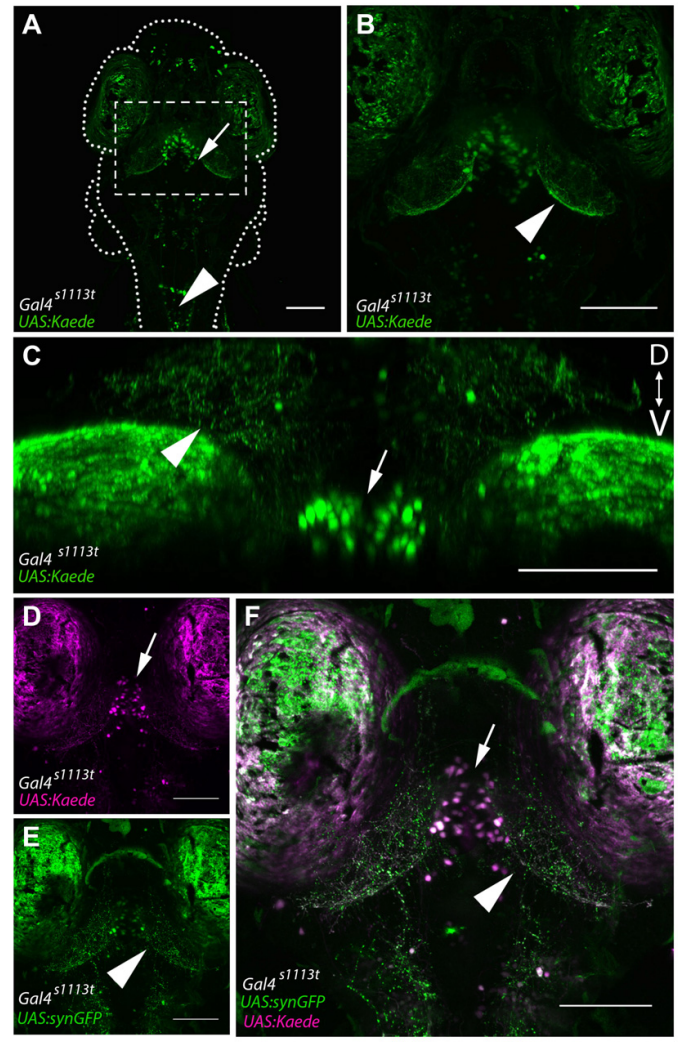

FIGURE 2 | Gal4-positive hypothalamic neurons project axons to the tectal neuropil. (A) Maximum projection of a $6 \mathrm{dpf}$ Gal4 ${ }^{\text {s1113t }}$;UAS:Kaede larva in which expression is strongest in a small number of neurons in the ventral diencephalon, located in the $\mathrm{RH}$ (arrow). (B) shows a closeup of the box in (A). Neurites are evident in the tectal neuropil (arrowhead) suggesting that hypothalamic projections may be targeting the tectum. (C) A coronal rotation through an animal with the genotype Gal4 ${ }^{\text {s1113t }}$;UAS:Kaede. The location of labeled cell bodies is indicated with an arrow, and projections to the tectal neuropil are labeled with an arrowhead. (D,E) Images from a

Gal4 ${ }^{\text {s1113t }}$;UAS:Kaede, UAS:syn-GFP showing the cell bodies of the $\mathrm{RH}$ neurons with photoconverted red Kaede (arrowhead, D) and the green presynaptic terminals of their axons in the tectal neuropil (arrow, E). These channels are merged in (F). Scale bars equal $100 \mu \mathrm{m}$.

and SAC/SPV (asterisks, Figures 3E,F). This shows that RH projection neurons target multiple discrete depths of the tectal neuropil, including both retinorecipient and non-retinorecipient laminae (Figure 3G).

\section{Functional Properties of Hypothalamic Inputs to the Tectum}

In order to gauge the functional relevance of these $\mathrm{RH}$ projections, we next observed the calcium responses that tectal neurons have to optogenetic $\mathrm{RH}$ stimulation. To do this, we used larvae with the genotype Gal4 $^{\text {s1113t }}$;UAS:Channelrhodopsin2(ET/TC)-mCherry, HuC:H2BGCaMP6s (Chen et al., 2013; Vladimirov et al., 2014). It is worth noting that in these experiments, the imaging plane was significantly more dorsal than the ChR2-expressing neurons in the RH. The result is that the illumination plane, although 
it is at $488 \mathrm{~nm}$, does not lead to any observable activation of ChR2-expressing neurons on the $\mathrm{RH}$.

To determine whether projections from the $\mathrm{RH}$ are excitatory or inhibitory in the tectum, we performed experiments where neurons expressing ChR2 were excited with a short (100 ms)
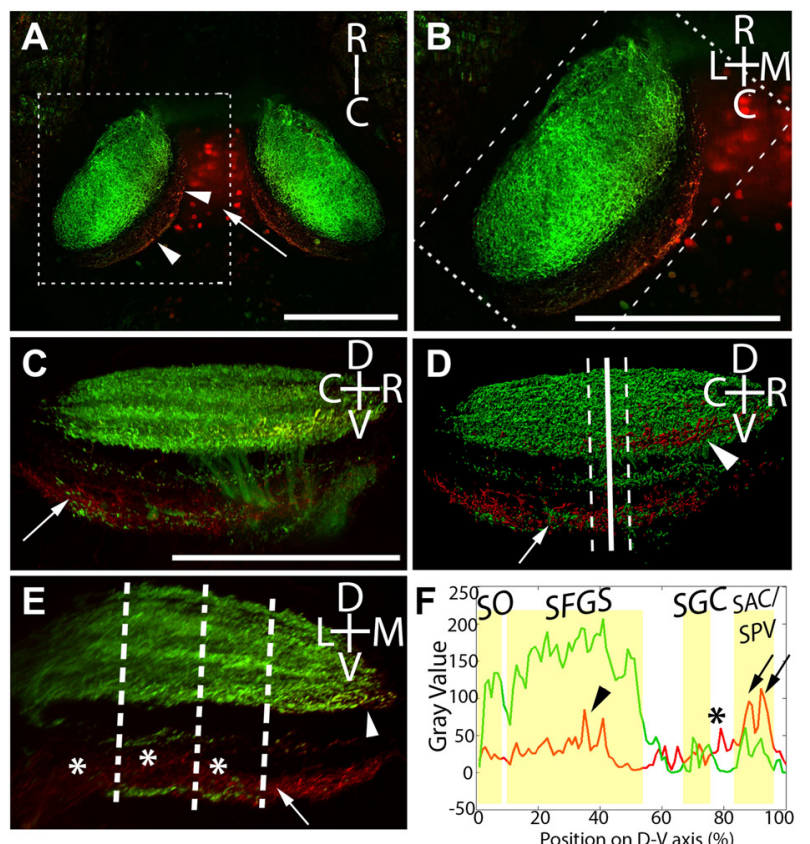

\section{G}

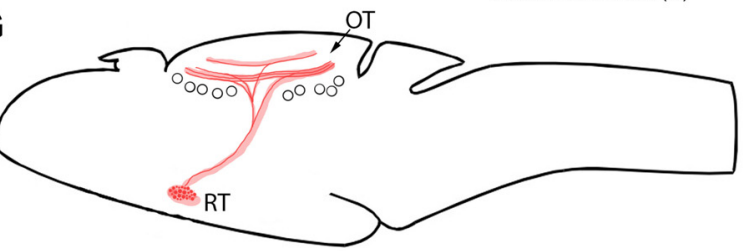

FIGURE 3 | Laminar structure of hypothalamic projections in the tectal neuropil. By expressing UAS:Kaede under the control of Atoh7:Gal4 and Gal4 ${ }^{\text {s1113t }}$, we have identified the neuropil laminae targeted by hypothalamic projection neurons. (A) Maximum intensity projection of a single larva at $20 x$ magnification, showing red photoconverted cells in the hypothalamus (arrow), as well as their neurites in the tectal neuropil (arrowheads). (B) An expanded view of the box in $(\mathbf{A})$, showing the relative positions of the retinal ganglion cells (RGC; green) and $\mathrm{RH}$ (red) axons. (C) A rotated view of the box in (B), with the medial edge of the neuropil perpendicular to the field of view. The arrow indicates hypothalamic axons in the stratum album centrale (SAC)/stratum griseum periventriculare (SPV). (D) An Imaris 3D rendering of (C). The solid line indicates the medial-lateral center point of the tectal neuropil, and dashed lines show the boundaries that were used in creating the coronal perspective in (E). The arrow indicates hypothalamic output to the SAC/SPV, arrowhead indicates hypothalamic output in the stratum fibrosum et griseum superficiale (SFGS). (E) A coronal perspective of the tectal neuropil labeling all laminae formed by the RGC axons (green) and hypothalamic inputs (red). To assess which sublaminae of the neuropil the hypothalamus targets, the fluorescent intensities of retinal and hypothalamic inputs were sampled at three evenly-spaced positions, indicated by dashed lines. (F) Hypothalamic inputs specifically targeted regions of the SFGS (arrowhead) and SAC/SPV (arrows) in the tectal neuropil. Additionally, hypothalamic projections frequently targeted a lamina devoid of retinal input between the stratum griseum centrale (SGC) and SAC/SPV (asterisks, E,F). Scale bar represents $100 \mu \mathrm{m}$.

(G) A schematic of the projection from the $\mathrm{RH}$ to the optic tectum in the Gal4s1113t transgenic line.
A

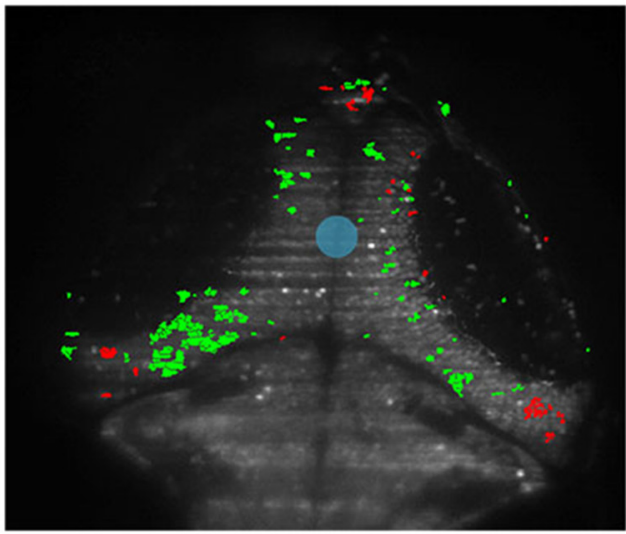

B

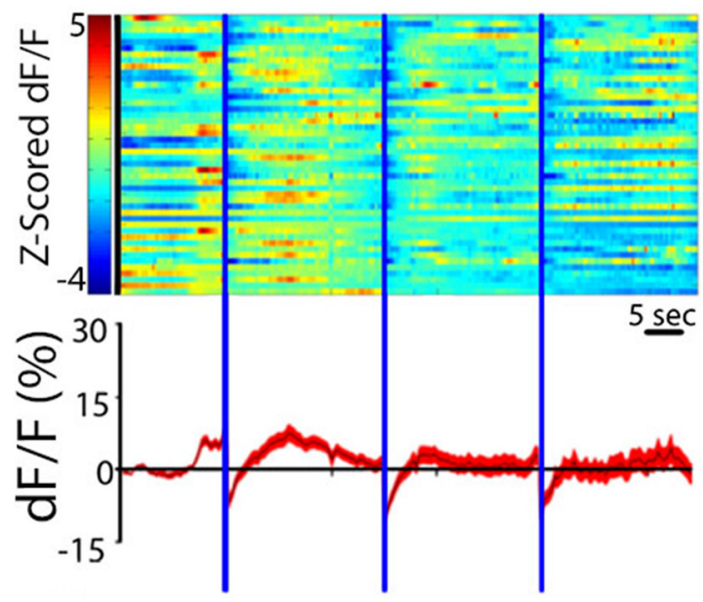

C

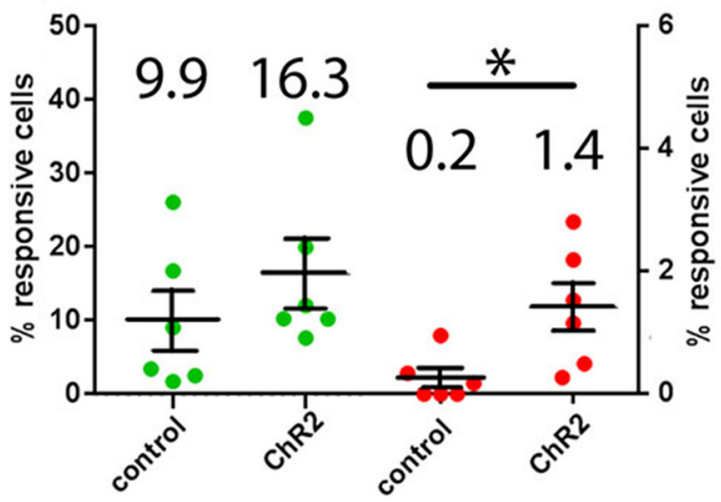

FIGURE 4 | Hypothalamic projection neurons deliver inhibitory inputs to the tectum. (A) Activation of ChR2-expressing neurons in the $\mathrm{RH}$ using sculpted blue light (shaded blue) results in inhibitory responses (shaded red) and excitatory visual responses (green) in tectal PVL neurons. (B) A raster plot of these responses ( $n=46$ inhibited neurons across six larvae) shows the $z$-scored $\Delta F / F$ of these inhibited neurons (top), and an average trace of these neurons through the experiment is shown (bottom). (C) Similar proportions of tectal cells were excited by a short pulse of blue light in $\mathrm{ChR2}{ }^{+}$larvae and ChR2 ${ }^{-}$controls, indicating that these are direct visual responses (green). Significantly more tectal neurons were inhibited in $\mathrm{ChR}^{+}$larvae vs. ChR2 ${ }^{-}$ controls (red) by a prolonged illumination of the $\mathrm{RH}$, indicating a causative effect of hypothalamic inputs. Dots represent individual larvae and mean \pm SEM is indicated, mean value is reported above each scatter plot. $* p<0.05$. $n=6$ experimental, $n=6$ controls. 
pulse of blue light. Such stimulation excited a similar number of tectal neurons both in our experimental larvae and in controls not expressing ChR2, suggesting that the responses in these experiments are simply tectal visual responses to the flash of light from the SLM (Figures $4 \mathbf{A}, \mathbf{C}$ ). As a means of probing for inhibitory effects, we extended our light pulse to $5 \mathrm{~s}$ in order to produce prolonged inhibition of postsynaptic tectal neurons. If $\mathrm{RH}$ inputs are inhibitory, this should result in a decrease in GCaMP signal in the postsynaptic cells (Tian et al., 2009; Akerboom et al., 2012), followed either by a return to baseline, or "rebound firing" in response to the disinhibition of the postsynaptic neurons at the end of hypothalamic stimulation (Bennett, 1966; Aizenman and Linden, 1999; Jay et al., 2015). Based on these expectations, a small but significant proportion of tectal neurons appeared to be inhibited by $\mathrm{RH}$ input (Figures 4B,C). These PVL neurons, both as individuals and as a population, showed decreased GCaMP fluorescence following the hypothalamic excitation, and in most cases, rebound firing. Habituation of this rebound firing occurred during the course of our three-trial experiment (Figure 4B).

\section{DISCUSSION}

\section{Nonretinal Projections to the Optic Tectum of Larval Zebrafish}

The tecta of various teleost species, including zebrafish, are known to receive input from the retina, and from a host of regions throughout the brain. Here, we demonstrate that neurons within the $\mathrm{RH}$ project to the tectum in larval zebrafish. These findings are similar to those described in numerous fish species including the hagfish and lamprey, where numerous hypothalamic clusters have been observed projecting in an ipsilateral fashion to the optic tectum (Amemiya, 1983; Meek and Nieuwenhuys, 1998; de Arriba and Pombal, 2007). In inspecting these projections in more detail, we also show that they specifically target the tectal neuropil's deeper retinorecipient laminae, as well as a non-retinorecipient lamina between the SGC and SAC/SPV. This arrangement has several implications for the overall structure of the tectal neuropil. It has previously been shown that the broad laminae delineated by RGC axons each comprise several sublaminae with distinct contributions to visual processing (Xiao and Baier, 2007; Bollmann and Engert, 2009; Xiao et al., 2011; Robles et al., 2013, 2014). The RH projections that we have found in retinorecipient laminae, especially the SFGS, occupy a sharp subset of the lamina, suggesting that they may be restricted to specific sublaminae. This suggests a tight coupling between retinal and nonretinal inputs to these laminae, and potentially specific contributions from the RH to particular types of visual processing. $\mathrm{RH}$ afferents also target one lamina, between the SGC and SAC/SPV, that is not innervated by RGCs. Two conclusions can be drawn from this observation. First, it shows that this non-retinorecipient lamina is not exclusively involved with secondary visual processing, since it is also incorporating distinct nonretinal inputs. Second, since $\mathrm{RH}$ inputs innervate a sharp subset of the space between the SGC and the SAC/SPV, it appears that these non-retinorecipient laminae, like their retinorecipient counterparts, may contain functionally distinct sublaminae.

\section{Functional Properties of the Tectum's Hypothalamic Inputs}

Following the anatomical descriptions of these $\mathrm{RH}$ projections to the tectum, it was important to determine their functional contributions to tectal circuitry. This involved both short pulses of optogenetic stimulation to the RH (designed to elicit excitatory responses in tectal PVL neurons) and long pulses of RH stimulation (designed to identify inhibited PVL neurons). Short pulses of excitation in the $\mathrm{RH}$ led to tectal activity, but this was not significantly higher in ChR expressing animals than in $\mathrm{ChR}^{-}$controls. This result is consistent with an absence of excitatory $\mathrm{RH}$ input to the tectum, or with a low level of excitatory input that fell below the sensitivity of our experiment. Long pulses of optogenetic stimulation to the $\mathrm{RH}$ drove inhibitory responses in the tectal PVL. The fact that there are apparently no tectal cells excited by $\mathrm{RH}$ stimulation suggests that the inhibited PVL neurons are directly post-synaptic to the projection neurons. Inhibition does not appear to result, for example, from the activation of tectal inhibitory neurons.

These experiments do not allow us to discriminate between the effects of direct monosynaptic connections and those resulting from more complex pathways. Therefore, it is possible that $\mathrm{RH}$ activity drives responses in an intermediate structure, which in turn inhibits PVL neurons. One candidate for such an intermediate structure would be the precursor to the preglomerular complex, the migrated posterior tubercular area (M2 region), which innervates the tectum (Mueller and Wullimann, 2005; Mueller, 2012). In the Gal4 ${ }^{\text {s1113t }}$ line, this structure appears to receive neurites from $\mathrm{RH}$ neurons expressing Gal4 (Figure 1I), supporting this possibility. Given the direct anatomical projection from the $\mathrm{RH}$ to the tectal neuropil in this line, however, the most parsimonious explanation is that the $\mathrm{RH}$ projection neurons are feeding directly into the tectal circuit.

\section{Implications for Tectal Processing}

The combined results of our anatomical and functional analyses have implications for the roles that nonretinal inputs may play in tectal processing. Given the diverse roles for the hypothalamus including arousal (Prober et al., 2006; Chiu and Prober, 2013), the detection of prey (Filosa et al., 2016) and feeding (Yokobori et al., 2011, 2012; Nishiguchi et al., 2012) it seems likely that these inhibitory signals may provide some sort of modulatory effect, conceivably influencing approach/escape decisions or other behavioral calculations being carried out by tectal circuits (Barker and Baier, 2015; Bianco and Engert, 2015). Muto et al. (2017) have recently shown that visual prey stimuli trigger hypothalamic activity in larval zebrafish, and that this information is relayed through the pretectum prior to arriving in the hypothalamus. Furthermore, expression of toxins in the hypothalamus reduced prey capture behavior. This 
raises a possible role for the hypothalamus in gating predatory behavior.

The anatomy of these projections appears to be similar with those described by Kaslin et al. (2004), who show that Orexin expression in the tectal neuropil is confined to the SAC in adult zebrafish. This finding suggests that orexinexpressing neurons within the hypothalamus of zebrafish may project to similar laminae of the optic tectum as the $\mathrm{RH}$ projections shown in this study. If our described projections are those described by Kaslin et al. (2004), it suggests that they could potentially be involved in controlling sleep and wakefulness (Kaslin et al., 2004). That the responses in the tectum are sparse and inhibitory further supports the idea that the hypothalamus' role is to influence or contextualize the sensory signals, rather than to be a major driver of them. Of course, the identity, morphology, and connectivity of the tectal cells inhibited by the RH will be of interest as this circuit is further explored, as would the activity of hypothalamic projection neurons in the contexts of predation, sleep and other behaviors.

More broadly, these results add to the direct evidence for a larval zebrafish tectum that receives more numerous and diverse inputs than had previously been recognized, more in line with what has been described in adult fish and tetrapods. The anatomical and circuit mapping experiments in this study compliment and extend recent work showing that the tectum and the Raphe, a structure known to target of hypothalamus in both larval and adult zebrafish (Lillesaar et al., 2009; Herculano and Maximino, 2014), work together to drive behaviors based on the feeding state of larvae (Filosa et al., 2016), and thus provides grounds to understand how the visual and metabolic systems work together to drive behaviors. Additionally, the results from the optogenetic studies presented here complement previous work describing the neurotransmitter profiles of neurons within the $\mathrm{RH}$, showing in this context that they have inhibitory effects on postsynaptic partners (Kaslin et al., 2004). Combined, the facts that the tectum receives direct afferents from several brain regions (Yokogawa et al., 2012; Heap et al., 2013; Filosa et al., 2016), and responds to multiple sensory modalities (Thompson et al., 2016), paint a picture of a structure that is more completely homologous to its mammalian counterpart, the superior colliculus, than has

\section{REFERENCES}

Aizenman, C. D., and Linden, D. J. (1999). Regulation of the rebound depolarization and spontaneous firing patterns of deep nuclear neurons in slices of rat cerebellum. J. Neurophysiol. 82, 1697-1709.

Akerboom, J., Chen, T.-W., Wardill, T. J., Tian, L., Marvin, J. S., Mutlu, S., et al. (2012). Optimization of a GCaMP calcium indicator for neural activity imaging. J. Neurosci. 32, 13819-13840. doi: 10.1523/JNEUROSCI.260112.2012

Amemiya, F. (1983). Afferent connections to the tectum mesencephali in the hagfish, Eptatretus burgeri: an HRP study. J. Hirnforsch. 24, 225-236.

Barker, A. J., and Baier, H. (2015). Sensorimotor decision making in the zebrafish tectum. Curr. Biol. 25, 2804-2814. doi: 10.1016/j.cub.2015. 09.055 previously been appreciated. They also suggest that the tectum may be less of a self-contained visual processing center, and more of an integrative locus for diverse information. While RGCs remain, both in anatomical and functional terms, the strongest single input to the tectum in larval zebrafish, it is increasingly clear that tectal function is broader and more nuanced than its traditional role as a visual processing center would suggest.

\section{AUTHOR CONTRIBUTIONS}

LAH designed experiments, collected and analyzed data and wrote the manuscript. GCV performed bioinformatic analyses of ontogenetics data. AWT created the UAS:syn-GFP line. IF-B and HR-D designed the sculpted light for optogenetics. IF-B performed these the experiments. EKS designed the experiment, interpreted the data and wrote the manuscript.

\section{ACKNOWLEDGMENTS}

We thank Rebecca Dunning for creating the PME-linker-MCS plasmid. We are grateful to Karl Deisseroth for providing the Channelrhodopsin-2(ET/TC) plasmid and Misha Ahrens for providing the $H u C: H 2 B-G C a M P 6 s$ transgenic line. We thank Owen Randlett, Alexander Schier, and Florian Engert for providing the Z-Brain atlas prior to publication, as well as for assisting in Z-Brain analysis. We thank Jan Kaslin and members of the Scott laboratory for comments on the manuscript. Support was provided by the Australian Research Council Postgraduate Awards to LAH and AWT; an National Health and Medical Research Council (NHMRC) Project Grant (APP1066887), ARC Future Fellowship (FT110100887), a Simons Foundation Explorer Award (336331) and two ARC Discovery Project Grants (DP140102036 and DP110103612) to EKS; an ARC Discovery Project (DP140100753) to HR-D; a UQ Postgraduate Scholarship to IF-B; and an European Molecular Biology Organization (EMBO) Long-term Fellowship (ALTF 727-2014) to GCV. Imaging was performed at the Queensland Brain Institute's Advanced Microscopy Facility, generously supported by the Australian Government through the ARC LIEF grant LE130100078.
Bennett, M. R. (1966). Rebound excitation of the smooth muscle cells of the guinea-pig taenia coli after stimulation of intramural inhibitory nerves. J. Physiol. 185, 124-131. doi: 10.1113/jphysiol.1966.sp007975

Berndt, A., Schoenenberger, P., Mattis, J., Tye, K. M., Deisseroth, K. Hegemann, P., et al. (2011). High-efficiency channelrhodopsins for fast neuronal stimulation at low light levels. Proc. Natl. Acad. Sci. U S A 108, 7595-7600. doi: 10.1073/pnas.1017210108

Best, J., Adatto, I., Cockington, J., James, A., and Lawrence, C. (2010). A novel method for rearing first-feeding larval zebrafish: polyculture with type L saltwater rotifers (Brachionus plicatilis). Zebrafish 7, 289-295. doi: 10.1089/zeb.2010.0667

Bianco, I. H., and Engert, F. (2015). Visuomotor transformations underlying hunting behavior in zebrafish. Curr. Biol. 25, 831-846. doi: 10.1016/j.cub.2015. 01.042 
Bolborea, M., and Dale, N. (2013). Hypothalamic tanycytes: potential roles in the control of feeding and energy balance. Trends Neurosci. 36, 91-100. doi: 10.1016/j.tins.2012.12.008

Bollmann, J. H., and Engert, F. (2009). Subcellular topography of visually driven dendritic activity in the vertebrate visual system. Neuron 61, 895-905. doi: 10.1016/j.neuron.2009.01.018

Chabot, N., Mellott, J. G., Hall, A. J., Tichenoff, E. L., and Lomber, S. G. (2013). Cerebral origins of the auditory projection to the superior colliculus of the cat. Hear. Res. 300, 33-45. doi: 10.1016/j.heares.2013.02.008

Chen, T. W., Wardill, T. J., Sun, Y., Pulver, S. R., Renninger, S. L., Baohan, A., et al. (2013). Ultrasensitive fluorescent proteins for imaging neuronal activity. Nature 499, 295-300. doi: 10.1038/nature12354

Chiu, C. N., and Prober, D. A. (2013). Regulation of zebrafish sleep and arousal states: current and prospective approaches. Front. Neural Circuits 7:58. doi: 10.3389/fncir.2013.00058

Corbo, C. P., Othman, N. A., Gutkin, M. C., Alonso, A. C., and Fulop, Z. L. (2012). Use of different morphological techniques to analyze the cellular composition of the adult zebrafish optic tectum. Microsc. Res. Tech. 75, 325-333. doi: 10.1002/jemt.21061

Crish, S. D., Comer, C. M., Marasco, P. D., and Catania, K. C. (2003). Somatosensation in the superior colliculus of the star-nosed mole. J. Comp. Neurol. 464, 415-425. doi: 10.1002/cne.10791

de Arriba, M. D. C., and Pombal, M. A. (2007). Afferent connections of the optic tectum in lampreys: an experimental study. Brain Behav. Evol. 69, 37-68. doi: $10.1159 / 000095272$

Del Bene, F., Wyart, C., Robles, E., Tran, A., Looger, L., Scott, E. K., et al. (2010). Filtering of visual information in the tectum by an identified neural circuit. Science 330, 669-673. doi: 10.1126/science.1192949

Dräger, U. C., and Hubel, D. H. (1976). Topography of visual and somatosensory projections to mouse superior colliculus. J. Neurophysiol. 39, 91-101. doi: 10.1152/jn.1976.39.1.91

Druga, R., and Syka, J. (1984). Projections from auditory structures to the superior colliculus in the rat. Neurosci. Lett. 45, 247-252. doi: 10.1016/03043940(84)90234-9

Dunn, T. W., Gebhardt, C., Naumann, E. A., Riegler, C., Ahrens, M. B., Engert, F., et al. (2016). Neural circuits underlying visually evoked escapes in larval zebrafish. Neuron 89, 613-628. doi: 10.1016/j.neuron.2015.12.021

Easter, S. S. Jr., and Nicola, G. N. (1996). The development of vision in the zebrafish (Danio rerio). Dev. Biol. 180, 646-663. doi: 10.1006/dbio.1996.0335

Fame, R. M., Brajon, C., and Ghysen, A. (2006). Second-order projections from the posterior lateral line in the early zebrafish brain. Neural Dev. 29, 1-4. doi: 10.1186/1749-8104-1-4

Favre-Bulle, I. A., Preece, D., Nieminen, T. A., Heap, L. A., Scott, E. K., and Rubinsztein-Dunlop, H. (2015). Scattering of sculpted light in intact brain tissue, with implications for optogenetics. Sci. Rep. 5:11501. doi: 10.1038/srep11501

Fernandes, A. M., Fero, K., Arrenberg, A. B., Bergeron, S. A., Driever, W., and Burgess, H. A. (2012). Deep brain photoreceptors control light-seeking behavior in zebrafish larvae. Curr. Biol. 22, 2042-2047. doi: 10.1016/j.cub.2012. 08.016

Fiebig, E., Ebbesson, S., and Meyer, D. (1983). Afferent connections of the optic tectum in the piranha (Serrasalmus nattereri). Cell Tissue Res. 231, 55-72. doi: $10.1007 / \mathrm{bf} 00215774$

Filosa, A., Barker, A. J., Dal Maschio, M., and Baier, H. (2016). Feeding state modulates behavioral choice and processing of prey stimuli in the zebrafish tectum. Neuron 90, 596-608. doi: 10.1016/j.neuron.2016. 03.014

Folgueira, M., Sueiro, C., Rodríguez-Moldez, I., Yáñez, J., and Anadón, R. (2007). Organization of the torus Longitudinalis in the rainbow trout (Oncorhynchus mykiss): an immunohistochemical study of the GABAergic system and a Dil tract-tracing study. J. Comp. Neurol. 503, 348-370. doi: 10.1002/cne. 21363

Gabriel, J. P., Trivedi, C. A., Maurer, C. M., Ryu, S., and Bollmann, J. H. (2012). Layer-specific targeting of direction-selective neurons in the zebrafish optic tectum. Neuron 76, 1147-1160. doi: 10.1016/j.neuron.2012.12.003

Gahtan, E., Tanger, P., and Baier, H. (2005). Visual prey capture in larval zebrafish is controlled by identified reticulospinal neurons downstream of the tectum. J. Neurosci. 25, 9294-9303. doi: 10.1523/jneurosci.2678-05.2005
Gandhi, N. J., and Katnani, H. A. (2011). Motor functions of the superior colliculus. Annu. Rev. Neurosci. 34, 205-231. doi: 10.1146/annurev-neuro061010-113728

Gerchberg, R. W., and Saxton, W. O. (1972). A practical algorithm for the determination of phase from image and diffraction plane pictures. Optik 35, 237-246.

Heap, L. A., Goh, C., Kassahn, K., and Scott, E. K. (2013). Cerebellar output in zebrafish: an analysis of spatial patterns and topography in eurydendroid cell projections. Front. Neural Circuits 7:53. doi: 10.3389/fncir.2013.00053

Herculano, A. M., and Maximino, C. (2014). Serotonergic modulation of zebrafish behavior: towards a paradox. Prog. Neuropsychopharmacol. Biol. Psychiatry 55, 50-66. doi: 10.1016/j.pnpbp.2014.03.008

Herget, U., Wolf, A., Wullimann, M. F., and Ryu, S. (2014). Molecular neuroanatomy and chemoarchitecture of the neurosecretory preoptichypothalamic area in zebrafish larvae. J. Comp. Neurol. 522, 1542-1564. doi: $10.1002 /$ cne. 23480

Hines, J. H., Ravanelli, A. M., Schwindt, R., Scott, E. K., and Appel, B. (2015). Neuronal activity biases axon selection for myelination in vivo. Nat. Neurosci. 18, 683-689. doi: 10.1038/nn.3992

Jay, M., De Faveri, F., and McDearmid, J. R. (2015). Firing dynamics and modulatory actions of supraspinal dopaminergic neurons during zebrafish locomotor behavior. Curr. Biol. 25, 435-444. doi: 10.1016/j.cub.2014. 12.033

Jay, M. F., and Sparks, D. L. (1987). Sensorimotor integration in the primate superior colliculus. I. Motor convergence. J. Neurophysiol. 57, 22-34.

Kaslin, J., Nystedt, J. M., Ostergård, M., Peitsaro, N., and Panula, P. (2004). The orexin/hypocretin system in zebrafish is connected to the aminergic and cholinergic systems. J. Neurosci. 24, 2678-2689. doi: 10.1523/jneurosci.490803.2004

King, A. J., Schnupp, J. W., Carlile, S., Smith, A. L., and Thompson, I. D. (1996). The development of topographically-aligned maps of visual and auditory space in the superior colliculus. Prog. Brain Res. 112, 335-350. doi: 10.1016/s00796123(08)63340-3

Kita, E. M., Scott, E. K., and Goodhill, G. J. (2015). Topographic wiring of the retinotectal connection in zebrafish. Dev. Neurobiol. 75, 542-556. doi: 10.1002/dneu.22256

Knudsen, E. I. (1982). Auditory and visual maps of space in the optic tectum of the owl. J. Neurosci. 2, 1177-1194.

Kokoeva, M. V., Yin, H., and Flier, J. S. (2005). Neurogenesis in the hypothalamus of adult mice: potential role in energy balance. Science 310, 679-683. doi: $10.1126 /$ science. 1115360

Krauzlis, R. J., Lovejoy, L. P., and Zénon, A. (2013). Superior colliculus and visual spatial attention. Annu. Rev. Neurosci. 36, 165-182. doi: 10.1146/annurevneuro-062012-170249

Kwan, K. M., Fujimoto, E., Grabher, C., Mangum, B. D., Hardy, M. E., Campbell, D. S., et al. (2007). The Tol2kit: a multisite gateway-based construction kit for Tol2 transposon transgenesis constructs. Dev. Dyn. 236, 3088-3099. doi: 10.1002/dvdy.21343

Lane, R. H., Allman, J. M., Kaas, J. H., and Miezin, F. M. (1973). The visuotopic organization of the superior colliculus of the owl monkey (Aotus trivirgatus) and the bush baby (Galago senegalensis). Brain Res. 60, 335-349. doi: 10.1016/0006-8993(73)90794-4

Lázár, G. (1973). The development of the optic tectum in Xenopus laevis: a Golgi study. J. Anat. 116, 347-355.

Legland, D., Arganda-Carreras, I., and Andrey, P. (2016). MorphoLibJ: integrated library and plugins for mathematical morphology with ImageJ. Bioinformatics 22, 3532-3534. doi: 10.1093/bioinformatics/btw413

Lillesaar, C., Stigloher, C., Tannhäuser, B., Wullimann, M. F., and Bally-Cuif, L. (2009). Axonal projections originating from raphe serotonergic neurons in the developing and adult zebrafish, Danio rerio, using transgenics to visualize raphe-specific pet1 expression. J. Comp. Neurol. 512, 158-182. doi: 10.1002/cne. 21887

Lister, J. A., Robertson, C. P., Lepage, T., Johnson, S. L., and Raible, D. W. (1999). nacre encodes a zebrafish microphthalmia-related protein that regulates neural-crest-derived pigment cell fate. Development 126, 3757-3767.

Lutz, C., Otis, T. S., DeSars, V., Charpak, S., DiGregorio, D. A., and Emiliani, V. (2008). Holographic photolysis of caged neurotransmitters. Nat. Methods 5, 821-827. doi: 10.1038/nmeth.1241 
May, P. J. (2006). The mammalian superior colliculus: laminar structure and connections. Prog. Brain Res. 151, 321-378. doi: 10.1016/s0079-6123(05) 51011-2

McPherson, A. D., Barrios, J. P., Luks-Morgan, S. J., Manfredi, J. P., Bonkowsky, J. L., Douglass, A. D., et al. (2016). Motor behavior mediated by continuously generated dopaminergic neurons in the zebrafish hypothalamus recovers after cell ablation. Curr. Biol. 26, 263-269. doi: 10.1016/j.cub. 2015.11.064

Meek, J. (1983). Functional anatomy of the tectum mesencephali of the goldfish. An explorative analysis of the functional implications of the laminar structural organization of the tectum. Brain Res. Rev. 6, 247-297. doi: 10.1016/01650173(83)90008-5

Meek, J., and Nieuwenhuys, R. (1998). "Holosteans and teleosts," in The Central Nervous System of Vertebrates, (Vol. 2) eds R. Nieuwenhuys, H. J. ten Donkelaar and C. Nicholson (Berlin: Springer), 759-937.

Meek, J., and Schellart, N. A. (1978). A golgi study of the goldfish optic tectum. J. Comp. Neurol. 182, 89-121. doi: 10.1002/cne.901820107

Meyer, F., and Beucher, S. (1990). Morphological segmentation. J. Vis. Commun. Image Represent. 1, 21-46. doi: 10.1016/1047-3203(90)90014-M

Mueller, T. (2012). What is the thalamus in zebrafish? Front. Neurosci. 6:64. doi: $10.3389 /$ fnins.2012.00064

Mueller, T., and Wullimann, M. (2005). Atlas of Early Zebrafish Brain Development: A Tool for Molecular Neurogenetics. Amsterdam: Elsevier.

Muto, A., Lal, P., Ailani, D., Abe, G., Itoh, M., and Kawakami, K. (2017). Activation of the hypothalamic feeding centre upon visual prey detection. Nat. Commun. 8:15029. doi: 10.1038/ncomms 15029

Nevin, L. M., Robles, E., Baier, H., and Scott, E. K. (2010). Focusing on optic tectum circuitry through the lens of genetics. BMC Biol. 8:126. doi: 10.1186/1741-7007 $-8-126$

Niell, C., and Smith, S. (2005). Functional imaging reveals rapid development of visual response properties in the zebrafish tectum. Neuron 45, 941-951. doi: 10.1016/j.neuron.2005.01.047

Nishiguchi, R., Azuma, M., Yokobori, E., Uchiyama, M., and Matsuda, K. (2012). Gonadotropin-releasing hormone 2 suppresses food intake in the zebrafish, Danio rerio. Front. Endocrinol. 3:122. doi: 10.3389/fendo.2012.00122

Northcutt, R. G. (1982). Localization of neurons afferent to the optic tectum in longnose gars. J. Comp. Neurol. 204, 325-335. doi: 10.1002/cne.902040404

Orger, M. B. (2016). The cellular organization of zebrafish visuomotor circuits. Curr. Biol. 26, R377-R385. doi: 10.1016/j.cub.2016.03.054

Perry, S. F., Ekker, M., Farrell, A. P., and Brauner, C. J. (2010). Fish Physiology: Zebrafish. Amerstand: Academic Press Elsevier.

Pityk, N. I. (1979). Hypothalamic influences on unit activity in the cat superior colliculus. Neurophysiology 11, 419-426. doi: 10.1007/bf01062837

Preibisch, S., Saalfeld, S., and Tomancak, P. (2009). Globally optimal stitching of tiled 3D microscopic image acquisitions. Bioinformatics 25, 1463-1465. doi: 10.1093/bioinformatics/btp184

Preuss, S. J., Trivedi, C. A., vom Berg-Maurer, C. M., Ryu, S., and Bollmann, J. H. (2014). Classification of object size in retinotectal microcircuits. Curr. Biol. 24, 2376-2385. doi: 10.1016/j.cub.2014.09.012

Prober, D. A., Rihel, J., Onah, A. A., Sung, R. J., and Schier, A. F. (2006). Hypocretin/orexin overexpression induces an insomnia-like phenotype in zebrafish. J. Neurosci. 26, 13400-13410. doi: 10.1523/JNEUROSCI.4332-06. 2006

Randlett, O., Wee, C. L., Naumann, E. A., Nnaemeka, O., Schoppik, D., Fitzgerald, J. E., et al. (2015). Whole-brain activity mapping onto a zebrafish brain atlas. Nat. Methods 12, 1039-1046. doi: 10.1038/nmeth. 3581

Rieck, R. W., Huerta, M. F., Harting, J. K., and Weber, J. T. (1986). Hypothalamic and ventral thalamic projections to the superior colliculus in the cat. J. Comp. Neurol. 243, 249-265. doi: 10.1002/cne.902430208

Robinson, D. L., and McClurkin, J. W. (1989). The visual superior colliculus and pulvinar. Rev. Oculomot. Res. 3, 337-360.

Robles, E., Filosa, A., and Baier, H. (2013). Precise lamination of retinal axons generates multiple parallel input pathways in the tectum. J. Neurosci. 33, 5027-5039. doi: 10.1523/JNEUROSCI.4990-12.2013

Robles, E., Laurell, E., and Baier, H. (2014). The retinal projectome reveals brainarea-specific visual representations generated by ganglion cell diversity. Curr. Biol. 24, 2085-2096. doi: 10.1016/j.cub.2014.07.080
Robles, E., Smith, S. J., and Baier, H. (2011). Characterization of genetically targeted neuron types in the zebrafish optic tectum. Front. Neural Circuits 5:1. doi: 10.3389/fncir.2011.00001

Sas, E., and Maler, L. (1986). The optic tectum of gymnotiform teleosts Eigenmannia virescens and Apteronotus leptorhynchus: a Golgi study. Neuroscience 18, 215-246. doi: 10.1016/0306-4522(86)90190-9

Scott, E. K., and Baier, H. (2009). The cellular architecture of the larval zebrafish tectum, as revealed by Gal4 enhancer trap lines. Front. Neural Circuits 3:13. doi: 10.3389/neuro.04.013.2009

Scott, E. K., Mason, L., Arrenberg, A. B., Ziv, L., Gosse, N. J., Xiao, T., et al. (2007). Targeting neural circuitry in zebrafish using GAL4 enhancer trapping. Nat. Methods 4, 323-326. doi: 10.1038/nmeth1033

Semmelhack, J. L., Donovan, J. C., Thiele, T. R., Kuehn, E., Laurell, E., and Baier, H. (2014). A dedicated visual pathway for prey detection in larval zebrafish. Elife 3:e04878. doi: 10.7554/eLife.04878

Smith, S. M., and Vale, W. W. (2006). The role of the hypothalamic-pituitaryadrenal axis in neuroendocrine responses to stress. Dialogues Clin. Neurosci. 8, 383-395.

Sparks, D. L. (1988). Neural cartography: sensory and motor maps in the superior colliculus. Brain Behav. Evol. 31, 49-56. doi: 10.1159/000116575

Sparks, D. L., and Hartwich-Young, R. (1989). The deep layers of the superior colliculus. Rev. Oculomot. Res. 3, 213-255.

Streidter, G., and Northcutt, R. (1989). Two distinct visual pathways through the superficial pretectum in a percomorph teleost. J. Comp. Neurol. 283, 342-354. doi: $10.1002 /$ cne. 902830304

Struermer, C. (1988). Retinotopic organization of the developing retinotectal projection in the zebrafish embryo. J. Neurosci. 8, 4513-4530.

Tay, T. L., Ronneberger, O., Ryu, S., Nitschke, R., and Driever, W. (2011). Comprehensive catecholaminergic projectome analysis reveals single-neuron integration of zebrafish ascending and descending dopaminergic systems. Nat. Commun. 2:171. doi: 10.1038/ncomms1171

Temizer, I., Donovan, J. C., Baier, H., and Semmelhack, J. L. (2015). A visual pathway for looming-evoked escape in larval zebrafish. Curr. Biol. 25, 1823-1834. doi: 10.1016/j.cub.2015.06.002

Thompson, A. W., Vanwalleghem, G. C., Heap, L. A., and Scott, E. K. (2016). Functional profiles of visual, auditory and water flow responsive neurons in the zebrafish tectum. Curr. Biol. 26, 743-754. doi: 10.1016/j.cub.2016.01.041

Tian, L., Hires, S. A., Mao, T., Huber, D., Chiappe, M. E., Chalasani, S. H., et al. (2009). Imaging neural activity in worms, flies and mice with improved GCaMP calcium indicators. Nat. Methods 6, 875-881. doi: 10.1038/ nmeth.1398

Udin, S. B. (2012). Binocular maps in Xenopus tectum: visual experience and the development of isthmotectal topography. Dev. Neurobiol. 72, 564-574. doi: 10.1002/dneu.20933

Ulrich-Lai, Y. M., and Herman, J. P. (2009). Neural regulation of endocrine and autonomic stress responses. Nat. Rev. Neurosci. 10, 397-409. doi: $10.1038 / \mathrm{nrn} 2647$

Vanegas, H., Laufer, M., and Amat, J. (1974). The optic tectum of a perciform teleost. I. General configuration and cytoarchitecture. J. Comp. Neurol. 154, 43-60. doi: 10.1002/cne.901540104

Vanwalleghem, G., Heap, L. A., and Scott, E. (2017). A profile of auditoryresponsive neurons in the larval zebrafish brain. J. Comp. Neurol. 525, 3031-3043. doi: 10.1002/cne.24258

Vladimirov, N., Mu, Y., Kawashima, T., Bennett, D. V., Yang, C. T., Looger, L. L., et al. (2014). Light-sheet functional imaging in fictively behaving zebrafish. Nat. Methods 11, 883-884. doi: 10.1038/nmeth.3040

Westerfield, M. (2000). The Zebrafish Book. A Guide for the Laboratory Use of Zebrafish (Danio rerio). 4th Edn. Eugene, OR: University of Oregon Press.

Whyte, G., and Courtial, J. (2005). Experimental demonstration of holographic three-dimensional light shaping using a Gerchberg-Saxton algorithm. New J. Phys. 7:117. doi: 10.1088/1367-2630/7/1/117

Withington-Wray, D. J., Binns, K. E., Dhanjal, S. S., Brickley, S. G., and Keating, M. J. (1990). The maturation of the superior collicular map of auditory space in the guinea pig is disrupted by developmental auditory deprivation. Eur. J. Neurosci. 2, 693-703. doi: 10.1111/j.1460-9568.1990.tb00459.x

Xiao, T., and Baier, H. (2007). Lamina-specific axonal projections in the zebrafish tectum require the type IV collagen Dragnet. Nat. Neurosci. 10, 1529-1537. doi: $10.1038 / \mathrm{nn} 2002$ 
Xiao, T., Staub, W., Robles, E., Gosse, N. J., Cole, G. J., and Baier, H. (2011). Assembly of lamina-specific neuronal connections by slit bound to type IV collagen. Cell 146, 164-176. doi: 10.1016/j.cell.2011.06.016

Xue, H.-G., Yamamoto, N., Yoshimoto, M., Yang, C.-Y., and Ito, H. (2001). Fiber connections of the nucleus isthmi in the carp (Cyprinus carpio) and tilapia (Oreochromis niloticus). Brain Behav. Evol. 58, 185-204. doi: 10.1159/000057563

Yokobori, E., Azuma, M., Nishiguchi, R., Kang, K. S., Kamijo, M., Uchiyama, M., et al. (2012). Neuropeptide $Y$ stimulates food intake in the Zebrafish, Danio rerio. J. Neuroendocrinol. 24, 766-773. doi: 10.1111/j.1365-2826.2012. 02281.x

Yokobori, E., Kojima, K., Azuma, M., Kang, K. S., Maejima, S., Uchiyama, M., et al. (2011). Stimulatory effect of intracerebroventricular administration of orexin A on food intake in the zebrafish, Danio rerio. Peptides 32, 1357-1362. doi: 10.1016/j.peptides.2011.05.010
Yokogawa, T., Hannan, M. C., and Burgess, H. A. (2012). The dorsal raphe modulates sensory responsiveness during arousal in zebrafish. J. Neurosci. 32, 15205-15215. doi: 10.1523/JNEUROSCI.1019-12.2012

Conflict of Interest Statement: The authors declare that the research was conducted in the absence of any commercial or financial relationships that could be construed as a potential conflict of interest.

Copyright (c) 2018 Heap, Vanwalleghem, Thompson, Favre-Bulle, RubinszteinDunlop and Scott. This is an open-access article distributed under the terms of the Creative Commons Attribution License (CC BY). The use, distribution or reproduction in other forums is permitted, provided the original author(s) or licensor are credited and that the original publication in this journal is cited, in accordance with accepted academic practice. No use, distribution or reproduction is permitted which does not comply with these terms. 